\title{
Do critically ill patients with AKI benefit from furosemide? Further real-word evidence from a large multi-center database
}

\author{
Shaowei Gao, Lu Yang and Zhongxing Wang*
}

We are interested in the recently published article about the effect of furosemide on the outcomes of patients with acute renal injury (AKI) [1], because the result seems against to the current guidelines [2].

AKI is commonly faced by the intensivists and its effects on mortality have drawn great attention recently. Zhao and his colleagues explored the effect of furosemide on the outcomes of critically ill patients with AKI in a real-word dataset, the MIMIC III database. They found the furosemide administration was associated with better short-term survival, especially in the $\mathrm{AKI}^{\mathrm{UO}}$ stages $2-3$. However, the dataset they used in the analysis is single-center.

We notice that another real-world database, the eICU database, which has larger size of data (200,859 patients) and more contributing centers (335 units at 208 hospitals), has been available to the world [3]. We reproduced the main analysis of Zhao's study in the eICU database to give more evidence for the real-world research. The main results for outcomes are presented in Table 1.

Some results are similar with Zhao's study [1]: the furosemide group has a higher recovery rate and longer hospital and ICU stay. However, a reduction in in- hospital mortality for the furosemide group only exists in the dataset after propensity score matching (PSM) and under the method of Cox regression (HR 0.92; 95\% CI $0.85-0.99 ; p=0.03$ ). For subgroup analysis, furosemide reduces mortality only in $\mathrm{AKI}^{\mathrm{SCR}}$ stages 2 and 3, but not in any of AKI or $\mathrm{AKI}^{\mathrm{UO}}$ stages.

Up to now, the main viewpoint is that diuretics including furosemide do not improve survival of AKI patients. The protective effect by improved renal function observed in some studies $[1,4]$ (include Zhao's and ours) may be covered by the adverse events, such as electrolyte abnormalities [5]. Our study supports no benefit for furosemide on in-hospital mortality of AKI patients, despite a significant $p$ value in the post-PSM dataset under the method of Cox regression (Table 1). Most of the studies use logistic regression to evaluate in-hospital mortality, because longer hospital stay for acute condition is not like longer follow-up time in chronic condition and may implicate bad outcomes. However, since the in-hospital mortality nearly doubles in the non-diuretic group comparing to the furosemide group in Zhao's study ( $21.7 \%$ vs $12.7 \%$ ), the debate of this issue still exists and needs more well-designed research.

* Correspondence: doctorwzx@126.com

Department of Anesthesia, the First Affiliated Hospital of Sun Yat-sen

University, 58 Zhongshan 2nd Road, Guangzhou 510080, China 
Table 1 Association between furosemide use and clinical outcomes in critically ill patients with acute kidney injury

\begin{tabular}{|c|c|c|c|c|}
\hline Outcomes & Non-diuretics & Furosemide & $p$ value & HR $(95 \% \mathrm{Cl})$ \\
\hline Pre-matched cohorts & 48,495 & 11,256 & & \\
\hline \multicolumn{5}{|l|}{ Primary } \\
\hline In-hospital mortality, n (\%) ${ }^{\mathrm{a}}$ & $5048(10.4)$ & $1327(11.8)$ & 0.18 & $0.96(0.90-1.02)$ \\
\hline In-hospital mortality, $n(\%)^{\mathrm{b}}$ & $5048(10.4)$ & $1327(11.8)$ & 0.98 & $1.00(0.93-1.07)$ \\
\hline \multicolumn{5}{|l|}{ Secondary } \\
\hline Recovery of renal function, $n(\%)^{\mathrm{b}}$ & $30,031(61.9)$ & $7105(63.1)$ & $<0.001$ & $1.10(1.05-1.14)$ \\
\hline Length of ICU stay, mean $(\mathrm{SD})^{c}$ & $4.1(5.0)$ & $4.7(5.2)$ & $<0.001$ & $1.53(1.35-1.73)$ \\
\hline Length of hospital stay, mean $(\mathrm{SD})^{c}$ & $10.3(9.0)$ & $11.1(8.7)$ & $<0.001$ & $1.46(1.18-1.80)$ \\
\hline Post-matched cohorts & 11,256 & 11,256 & & \\
\hline \multicolumn{5}{|l|}{ Primary } \\
\hline In-hospital mortality, n (\%) ${ }^{\mathrm{a}}$ & $1415(12.6)$ & $1327(11.8)$ & 0.03 & $0.92(0.85-0.99)$ \\
\hline In-hospital mortality, $n(\%)^{\mathrm{b}}$ & $1415(12.6)$ & $1327(11.8)$ & 0.49 & $0.97(0.89-1.06)$ \\
\hline \multicolumn{5}{|l|}{ Secondary } \\
\hline Recovery of renal function, $n(\%)^{\mathrm{b}}$ & $6791(60.3)$ & $7105(63.1)$ & $<0.001$ & $1.12(1.06-1.19)$ \\
\hline Length of ICU stay, mean $(\mathrm{SD})^{c}$ & $4.3(4.8)$ & $4.7(5.2)$ & $<0.001$ & $1.41(1.20-1.65)$ \\
\hline Length of hospital stay, mean $(S D)^{c}$ & $10.7(8.9)$ & $11.1(8.7)$ & $<0.001$ & $1.55(1.19-2.00)$ \\
\hline
\end{tabular}

${ }^{a}$ Cox proportional hazard regression was used to adjust the confounding variables

bLogistic regression was used to adjust the confounding variables

'Linear regression was used to adjust the confounding variables

The confounding variables include age, gender, ethnicity, admission type, comorbidities, mechanical ventilation use, vasopressor use, fluid balance first day, serum creatinine, eGFR, and APCHE IV predicted hospital mortality. Sensitivity analysis was conducted by selecting a part of all variables by clinical experts, and the $p$ value never changed from $<0.05$ to $>0.05$ or from $>0.05$ to $<0.05$, which presented robust

Abbreviations: HR hazard ratio, $\mathrm{Cl}$ confidence interval, ICU intensive care unit, SD standard deviation

\section{Authors' response}

Response to the letter entitled "Do critically ill patients

with AKI benefit from furosemide? Further real-word

evidence from a large multi-center database"

Guang-ju Zhao, Chang Xu, Zhong-qiu Lu

We appreciate Dr. Wang and his colleagues for their comments on our recent article related to the association between furosemide use and outcomes in critically ill patients with AKI [1]. Using the eICU database, they found that furosemide use was associated with increased renal function recovery rate but not reduced in-hospital mortality. MIMIC III is a single-center database which contains ICU patient data between 2001 and 2012, while the eICU database covers patients who were admitted to 208 centers in 2014 and 2015. The inconsistent results may be due to the different treatment strategies of AKI in the two periods. Nevertheless, many previous studies also showed conflicting results, the potential reasons for this is worth exploring.

First, the protective effect of diuretic on AKI is at least partly mediated by fluid balance $[1,6]$. So, the diversities of volume status among different AKI cohorts need to be considered when interpreting the conflicting results. Second, it has been noticed that the association between increased risk of death and furosemide use was more frequently reported in cohorts with higher SCr. So, in addition to the increased levels of $\mathrm{SCr}$ to baseline, the values of $\mathrm{SCr}$ and eGFR may also contribute to differences in treatment outcomes. Finally, the start time of diuretics may also determine the effect of them on the outcomes of AKI. In our cohort, there were 1591 AKI patients who received furosemide treatment $48 \mathrm{~h}$ after admission, and the mortality of them was similar to those without diuretic treatment $(22.3 \%$ versus $21.7 \%$, $p>0.05)$.

Recently, more and more new sub-classes of AKI with different clinical profiles, including mortality, the speed of renal function recovery, and fluid and furosemide responsiveness, have been recognized [7-9]. In selected 
AKI patients, some real-world studies (including Dr. Wang's and ours) and multi-center prospective cohort studies have illustrated that furosemide use was associated with improved renal function recovery rate and (or) reduced mortality. Nevertheless, further high-quality study is needed to identify the sub-classes of AKI that can benefit from furosemide treatment.

\section{Abbreviations}

AKI: Acute renal injury; PSM: Propensity score match; ICU: Intensive care unit; HR: Hazard ratio; $\mathrm{Cl}$ : Confidence interval

\section{Acknowledgements}

We appreciate the researchers at the MIT Laboratory for Computational Physiology for publicly sharing the clinical databases of elCU.

\section{Funding}

The authors received no funding for this study.

\section{Availability of data and materials}

The datasets generated and/or analyzed during the current study are available in the elCU database (https://eicu-crd.mit.edu/).

\section{Ethics approval and consent to participate}

Not applicable

\section{Consent for publication}

Not applicable

Received: 31 March 2020 Accepted: 17 April 2020

Published online: 25 May 2020

\section{References}

1. Zhao G-J, Xu C, Ying J-C, Lü W-B, Hong G-L, Li M-F, Wu B, Yao Y-M, Lu Z-Q. Association between furosemide administration and outcomes in critically ill patients with acute kidney injury. Crit Care. 2020;24(1):75.

2. Kellum JA, Lameire N, Aspelin P, Barsoum RS, Burdmann EA, Goldstein SL, Herzog CA, Joannidis M, Kribben A, Levey AS, et al. Kidney disease: Improving global outcomes (KDIGO) acute kidney injury work group. KDIGO clinical practice guideline for acute kidney injury. Kidney Int Suppl. 2012;2(1): $1-138$.

3. Pollard TJ, Johnson AEW, Raffa JD, Celi LA, Mark RG, Badawi O. The elCU Collaborative Research Database, a freely available multi-center database for critical care research. Sci Data. 2018;5:180178.

4. Cantarovich F, Galli C, Benedetti L, Chena C, Castro L, Correa C, Perez Loredo J, Fernandez JC, Locatelli A, Tizado J. High dose frusemide in established acute renal failure. Br Med J. 1973;4(5890):449-50.

5. Bagshaw SM, Gibney RTN, Kruger P, Hassan I, McAlister FA, Bellomo R. The effect of low-dose furosemide in critically ill patients with early acute kidney injury: a pilot randomized blinded controlled trial (the SPARK study). J Crit Care. 2017;42:138-46.

6. Grams ME, Estrella MM, Coresh J, Brower RG, Liu KD, National Heart, Lung, and Blood Institute Acute Respiratory Distress Syndrome Network. Fluid balance, diuretic use, and mortality in acute kidney injury. Clin J Am Soc Nephrol. 2011;6:966-73.

7. Perinel S, Vincent F, Lautrette A, Dellamonica J, Mariat C, Zeni F, et al. Transient and persistent acute kidney injury and the risk of hospital mortality in critically ill patients: results of a multicenter cohort study. Crit Care Med. 2015;43:e269-75.

8. Zhang Z, Ho KM, Hong Y. Machine learning for the prediction of volume responsiveness in patients with oliguric acute kidney injury in critical care. Crit Care. 2019;23:112.

9. Matsuura R, Komaru Y, Miyamoto $Y$, Yoshida T, Yoshimoto K, Isshiki R, et al. Response to different furosemide doses predicts AKI progression in ICU patients with elevated plasma NGAL levels. Ann Intensive Care. 2018;8:8.

\section{Publisher's Note}

Springer Nature remains neutral with regard to jurisdictional claims in published maps and institutional affiliations. 